\title{
Habituation of the responsiveness of mesolimbic and mesocortical dopamine transmission to taste stimuli
}

\author{
Maria A. De Luca ${ }^{1,2} *$ \\ ${ }^{1}$ Department of Biomedical Sciences, Neuropsychopharmacology Section, University of Cagliari, Cagliari, Italy \\ ${ }^{2}$ National Institute of Neuroscience, University of Cagliari, Cagliari, Italy
}

\section{Edited by:}

Susanne Schmid, University of Western Ontario, Canada

\section{Reviewed by:}

Ranier Gutierrez, Centro de Investigación y de Estudios Avanzados del Instituto Politécnico Nacional, Mexico

Steven R. Laviolette, University of Western Ontario, Canada

James Edgar McCutcheon, University of Leicester, UK

\section{${ }^{*}$ Correspondence:}

Maria A. De Luca, Department of Biomedical Sciences,

Neuropsychopharmacology Section, University of Cagliari, Via Ospedale 72, 09124 Cagliari, Italy e-mail:deluca@unica.it
The presentation of novel, remarkable, and unpredictable tastes increases dopamine (DA) transmission in different DA terminal areas such as the nucleus accumbens (NAc) shell and core and the medial prefrontal cortex (MPFC), as estimated by in vivo microdialysis studies in rats. This effect undergoes adaptive regulation, as there is a decrease in DA responsiveness after a single pre-exposure to the same taste. This phenomenon termed habituation has been described as peculiar to NAc shell but not to NAc core and mPFC DA transmission. On this basis, it has been proposed that MPFC DA codes for generic motivational stimulus value and, together with the NAc core DA, is more consistent with a role in the expression of motivation. Conversely, NAc shell DA is specifically activated by unfamiliar or novel taste stimuli and rewards, and might serve to associate the sensory properties of the rewarding stimulus with its biological effect (Bassareo etal., 2002; Di Chiara et al., 2004). Notably, habituation of the DA response to intraoral sweet or bitter tastes is not associated with a reduction in hedonic or aversive taste reactions, thus indicating that habituation is unrelated to satiety-induced hedonic devaluation and that it is not influenced by DA alteration or depletion. This mini-review describes specific circumstances of disruption of the habituation of NAc shell DA responsiveness (De Luca etal., 2011; Bimpisidis etal., 2013). In particular, we observed an abolishment of NAc shell DA habituation to chocolate (sweet taste) by morphine sensitization and mPFC 6hydroxy-dopamine hydrochloride (6-OHDA) lesion. Moreover, morphine sensitization was associated with the appearance of the habituation in the MPFC, and with an increased and delayed response of NAc core DA to taste in naive rats, but not in pre-exposed animals. The results here described shed light on the mechanism of the habituation phenomenon of mesolimbic and mesocortical DA transmission, and its putative role as a marker of cortical dysfunction in specific conditions such as addiction.

Keywords: habituation, dopamine, nucleus accumbens, medial prefrontal cortex, taste stimuli, microdialysis

\section{INTRODUCTION}

Primary motivational states, both positive and negative, are often ruled by the activity of dopamine (DA) neurons in the ventral tegmental area (VTA) and their terminal targets, such as the nucleus accumbens (NAc) and the medial prefrontal cortex (mPFC). In these terminal regions, DA responds to appetitive or aversive stimuli differently depending on specific factors such as stimulus valence, stimulus sensory modality, specific DA neuron subpopulations, different terminal areas studied, and the techniques used for the detection of DA (e.g., microdialysis vs voltammetry; Fibiger and Phillips, 1988; Di Chiara, 1995; Westerink, 1995; Berridge and Robinson, 1998; Schultz, 1998; Redgrave et al., 1999; Di Chiara et al., 2004; Aragona et al., 2009; Lammel et al., 2012; McCutcheon et al., 2012).

The direct correlation between motivational stimulus valence and its effect on the responsiveness of DA transmission has been

\footnotetext{
Abbreviation: C, chocolate; DA, dopamine; i.o., intraorally; $\mathrm{mPFC}$, medial prefrontal cortex; NAc, nucleus accumbens; 6-OHDA, 6-hydroxy-dopamine hydrochloride; s.c., subcutaneously; VTA, ventral tegmental area.
}

extensively appreciated by in vivo brain microdialysis studies in three different DA terminal areas: NAc shell, NAc core, and mPFC (Bassareo and Di Chiara, 1999; Bassareo et al., 2002). Particularly, it has been observed that the exposure to natural rewards (e.g., highly palatable food) and to salient food taste stimuli (sweet and bitter) increases DA transmission in NAc shell and core and in mPFC of non-food-deprived rats. In NAc shell, but not in NAc core or in $\mathrm{mPFC}$, this response undergoes adaptive regulation after a single pre-exposure to the same taste/food. This response reduces following a recurrent stimulus, and is termed habituation (Thompson and Spencer, 1966; Cohen et al., 1997; Rankin et al., 2009). In NAc shell, habituation to natural rewards is taste specific, and it is reversed by food deprivation of the animals and modified by the presentation of cues associated with the stimulus (Bassareo and Di Chiara, 1999). These observations demonstrate that NAc shell DA is activated by unfamiliar appetitive taste stimuli while DA in the mPFC codes for generic motivational value independently of stimulus valence. Additionally, this underlines the role of NAc shell DA and its habituation in associative learning (Bassareo et al., 2002; Di Chiara et al., 2004). 
In contrast, habituation of DA response is not present after repeated exposure to drugs of abuse (e.g., nicotine, opiates, psychostimulants, cannabinoids), which preferentially stimulate DA transmission in NAc shell as compared to NAc core (Pontieri et al., 1995, 1996; Tanda et al., 1997). However, the use of in vivo voltammetry by other labs showed opposite and specific sub-regional changes in DA concentration in response to both cued and unconditioned appetitive stimuli or after cocaine (Aragona et al., 2009; Brown et al., 2011; Badrinarayan et al., 2012).

This review describes experimental evidence for the disruption of habituation of NAc shell DA responsiveness to motivational stimuli in vivo, and on the specific circumstances that could contribute to these significant changes. The data here discussed highlight the role of DA in both learning and hedonic processes.

\section{SENSITIZATION TO MORPHINE AFFECTS HABITUATION OF MESOLIMBIC AND MESOCORTICAL DOPAMINE RESPONSIVENESS TO TASTE STIMULI}

Morphine administration increases DA transmission in the mesolimbic system, as estimated by in vivo brain microdialysis (Di Chiara and Imperato, 1988; Pontieri et al., 1996). Specific experimental protocols of repeated exposure to morphine produced sensitization.

The effect of morphine sensitization on the habituation of the responsiveness of DA transmission to a single pre-exposure to novel, remarkable and unpredictable taste stimuli has been evaluated (De Luca et al., 2011). In order to induce behavioral and biochemical sensitization, a protocol conceived by Cadoni and Di Chiara (1999) has been used. Thus, rats were administered twice a day for three consecutive days with increasing doses of morphine $(10,20,40 \mathrm{mg} / \mathrm{kg}$ s.c. $)$ or saline. After 15 days of withdrawal, rats were administered a precise amount of appetitive sweet chocolate solution through an intraoral cannula ( $1 \mathrm{ml} / 5 \mathrm{~min}$, i.o.) during the microdialysis session for NAc shell, core and mPFC dialysate DA analysis.

Our main finding was that opiate sensitization and chocolate pre-exposure exert a differential influence on the response of DA transmission as regards to the specific subdivision of the mesocorticolimbic DA system. Figure 1 shows the effect of morphine sensitization on the response of NAc shell and core and mPFC DA levels to intraoral sweet chocolate in naive and chocolate pre-exposed rats. We reported that pre-exposure to chocolate produced opposite changes in DA transmission in the $\mathrm{mPFC}$ and in the NAc shell (De Luca et al., 2011). In fact, unexpected appearance of habituation in $\mathrm{mPFC}$ DA responsiveness to taste stimuli was accompanied by a loss of habituation in NAc shell. Moreover, morphine sensitization was associated with an increased and delayed (50-110 min after chocolate) response of NAc core DA to taste in naive rats while an immediate increase of DA was observed in pre-exposed animals. Similar results were obtained with an aversive stimulus (De Luca et al., 2011). Moreover, although sensitization to morphine is associated with long-term changes in mesolimbic and mesocortical DA responsiveness to taste stimuli, changes in behavioral taste reactivity are lacking. The latter evidence supports the hypothesis that taste-hedonia does not depend on DA (Berridge and Robinson, 1998), thus the increase of DA

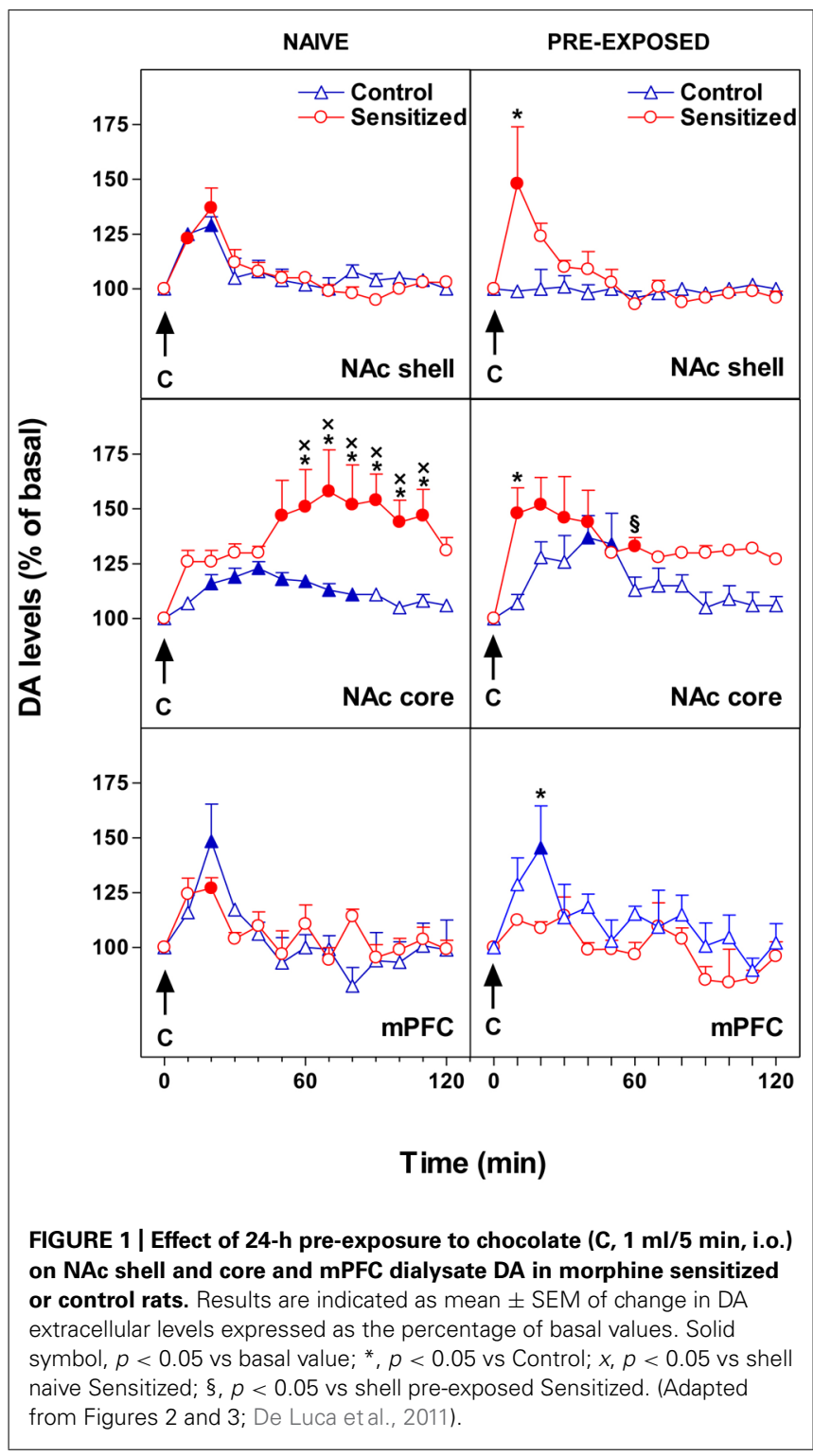

transmission in these brain regions could arise from the motivational and not from the sensory or hedonic properties of the taste (Bassareo and Di Chiara, 1999; Bassareo et al., 2002).

All of the DA terminal regions studied displayed changes in the habituation (i.e., abolishment vs appearance), which might result in an increased incentive arousal and learning. Notably, the habituation of $\mathrm{mPFC}$ DA responsiveness to chocolate releases NAc shell DA from inhibition, thereby abolishing the single-trial habituation of DA. Under this condition, repeated approaches toward a motivational stimulus might be facilitated.

\section{THE ABLATION OF THE mPFC DOPAMINE TERMINALS AFFECTS HABITUATION OF MESOLIMBIC DOPAMINE RESPONSIVENESS TO TASTE STIMULI}

In intact brain, $\mathrm{mPFC}$ DA prominently regulates the activity of subcortical DA areas involved in reward and motivation through a complex interaction of many different sub-regions 
inside the PFC (Murase et al., 1993; Taber and Fibiger, 1995; Kennerley and Walton, 2011). Such control is modulated by DA receptors in the mPFC (Louilot et al., 1989; Jaskiw et al., 1991; Vezina etal., 1991; Lacroix etal., 2000). mPFC DA functions are engaged in cognitive processes (Seamans and Yang, 2004), regulation of emotions (Sullivan, 2004), working memory (Khan and Muly, 2011), and executive functions such as motor planning, inhibitory response control and sustained attention (Fibiger and Phillips, 1988; Granon et al., 2000; Robbins, 2002).

We recently studied the effect of mPFC 6-OHDA lesion on NAc shell and core DA responsiveness to chocolate in naive and chocolate pre-exposed rats. 6-OHDA bilateral infusions in the mPFC modify the responsiveness of NAc DA to gustatory stimuli administered by an intraoral catheter. As shown in Figure 2, we observed that in NAc shell of naive subjects the lesion did not change the DA response to intraoral chocolate. However, the lesion of mPFC DA terminals produced an elevated, delayed, and prolonged increase of DA in NAc core in response to an appetitive taste stimulus. In pre-exposed subjects, the lesion did not affect NAc core DA responsiveness to chocolate while it abolished one-trial habituation of NAc shell DA response to sweet taste. After DA terminal lesions, an effect on neither hedonic taste score nor motor activity has been observed (Bimpisidis et al., 2013).

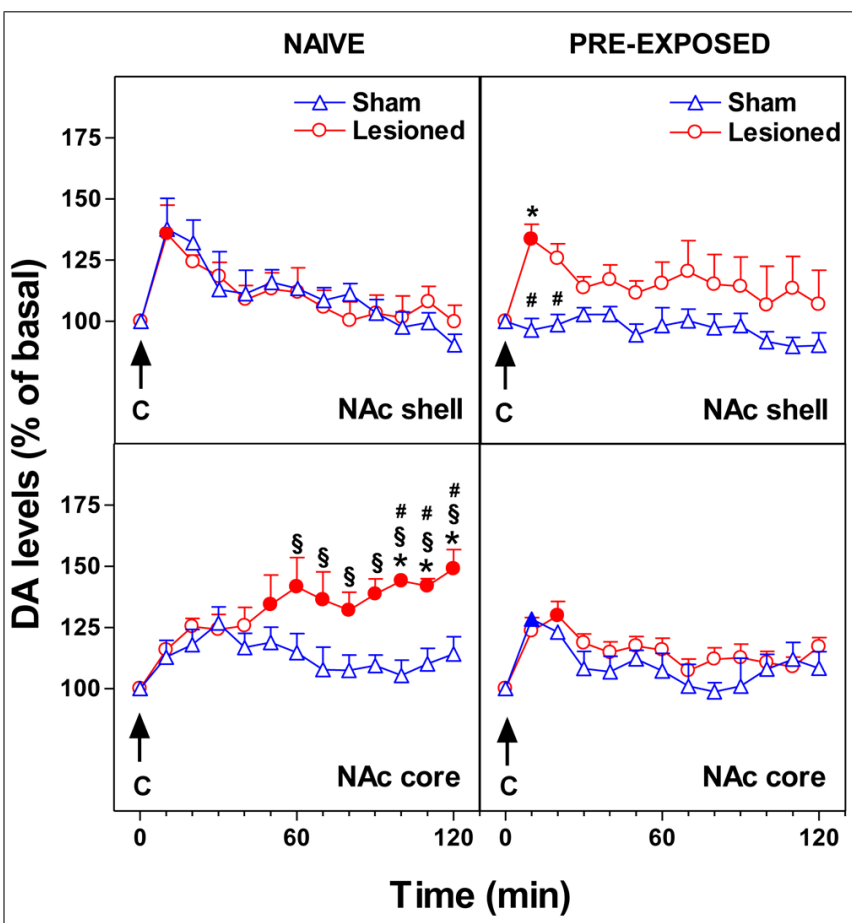

FIGURE 2 | Effect of 24-h pre-exposure to chocolate (C, $1 \mathrm{ml} / 5 \mathrm{~min}$, i.o.) on NAc shell and core dialysate DA in 6-OHDA lesioned in the MPFC or control rats. Results are indicated as mean \pm SEM of change in DA extracellular levels expressed as the percentage of basal values. Solid symbol, $p<0.05$ vs basal value; ${ }^{*}, p<0.05$ vs Sham; $\#, p<0.05$ vs Lesioned pre-exposed; $\S, p<0.05$ vs Sham pre-exposed. (Adapted from Figure 6; Bimpisidis et al., 2013).
These observations might suggest that the mPFC DA inhibitory control of DA responsiveness in subcortical striatal areas is different depending on the ventral striatum sub-region studied. Moreover, different sub-regions within the mPFC (e.g., prelimbic, infralimbic) have different projections to different compartments of the NAc. Accordingly, in the NAc shell, which is mostly innervated by the infralimbic area, the cortical-subcortical relationship might work in an opposite manner to that in NAc core.

This is consistent with the different responsiveness of NAc shell and core DA to discrete stimuli and conditions (Di Chiara et al., 2004; Di Chiara and Bassareo, 2007; Aragona et al., 2009; Corbit and Balleine, 2011; Cacciapaglia et al., 2012).

\section{CONCLUSION}

The experimental results here described may help explain, in part, the reason why traumatic PFC injury often facilitates development of drug use disorders (Delmonico et al., 1998). Accordingly, disruption of PFC functions appears following both traumatic conditions (Bechara and Van Der Linden, 2005) and history of drug addiction (Van den Oever et al., 2010; Goldstein and Volkow, 2011). Our data also suggest a correlation between the NAc DA responsiveness to repeated exposure to a motivational stimulus and the control of its activity by the mPFC DA. This refers to $\mathrm{mPFC}$ a crucial role in subcortical dysfunction, which may occur in different stages of drug addiction. Similarly, the mPFC plays a crucial role in subcortical dysfunction, which may occur in different stages of drug addiction. Other studies show the direct involvement of mPFC in addiction (Schenk et al., 1991; Weissenborn et al., 1997; Bolla et al., 2003), drug seeking, craving and relapse, which are related to drugs taken either by humans or animals (Kalivas and Volkow, 2005).

Remarkably, we found similarities between the effect of repeated morphine exposure and selective mPFC DA terminal lesions on DA transmission in response to motivational taste stimuli both in NAc shell and in NAc core. However, this correlation seems to exist only after prolonged administration of drugs of abuse, as a single drug exposure did not modify the habituation in NAc shell (De Luca et al., 2012). Moreover, the absence of any relationship between DA habituation and taste reactivity (Berridge, 2000; Bassareo et al., 2002; De Luca et al., 2012) has been validated.

In summary, the specific conditions leading to the abolishment of habituation illustrated in this work clarify the meaning of the habituation phenomenon of mesolimbic and mesocortical DA transmission. Habituation is usually present in NAc shell, but not in NAc core or mPFC, and it is ruled by intact DA transmission within the mPFC. However, the appearance of habituation in the mPFC could be considered as a marker of mPFC dysfunction in its ability to inhibit crucial subcortical functions. This may result in excessive motivation for inappropriate actions originating from a clear loss of impulse control. Finally, yet importantly, NAc DA habituation may be considered per se as a marker of drug dependence and its liability.

\section{ACKNOWLEDGMENTS}

This work was supported by grant from the Fondazione Banco di Sardegna, and by RAS LR 7, 2007. The author would like to 
thank Ms. Tonka Ivanisevic for the help with the preparation of the manuscript.

\section{REFERENCES}

Aragona, B. J., Day, J. J., Roitman, M. F., Cleaveland, N. A., Wightman, R. M., and Carelli, R. M. (2009). Regional specificity in the real-time development of phasic dopamine transmission patterns during acquisition of a cue-cocaine association in rats. Eur. J. Neurosci. 30, 1889-1899. doi: 10.1111/j.1460-9568.2009.07027.x

Badrinarayan, A., Wescott, S. A., Vander Weele, C. M., Saunders, B. T., Couturier, B. E., Maren, S., et al. (2012). Aversive stimuli differentially modulate real-time dopamine transmission dynamics within the nucleus accumbens core and shell. J. Neurosci. 7, 15779-15790. doi: 10.1523/JNEUROSCI. 3557-12.2012

Bassareo, V., De Luca, M. A., and Di Chiara, G. (2002). Differential expression of motivational stimulus properties by dopamine in nucleus accumbens shell versus core and prefrontal cortex. J. Neurosci. 22, 4709-4719.

Bassareo, V., and Di Chiara, G. (1999). Modulation of feeding-induced activation of mesolimbic dopamine transmission by appetitive stimuli and its relation to motivational state. Eur. J. Neurosci. 11, 4389-4397. doi: 10.1046/j.14609568.1999.00843.x

Bechara A., and Van Der Linden, M. (2005). Decision-making and impulse control after frontal lobe injuries. Curr. Opin. Neurol. 18, 734-739. doi: 10.1097/01.wco.0000194141.56429.3c

Berridge, K. C. (2000). Measuring hedonic impact in animals and infants: microstructure of affective taste reactivity patterns. Neurosci. Biobehav. Rev. 24, 173-198. doi: 10.1016/S0149-7634(99)00072-X

Berridge, K. C., and Robinson, T. E. (1998). What is the role of dopamine in reward: hedonic impact, reward learning, or incentive salience? Brain Res. Brain Res. Rev. 28, 309-369. doi: 10.1016/S0165-0173(98)00019-8

Bimpisidis, Z., De Luca, M. A., Pisanu, A., and Di Chiara, G. (2013). Lesion of medial prefrontal dopamine terminals abolishes habituation of accumbens shell dopamine responsiveness to taste stimuli. Eur. J. Neurosci. 37, 613-622. doi: 10.1111/ejn. 12068

Bolla, K. I., Eldreth, D. A., London, E. D., Kiehl, K. A., Mouratidis, M., Contoreggi, C., et al. (2003). Orbitofrontal cortex dysfunction in abstinent cocaine abusers performing a decision-making task. Neuroimage 19, 1085-1094. doi: 10.1016/S1053-8119(03)00113-7

Brown, H. D., McCutcheon, J. E., Cone, J. J., Ragozzino, M. E., and Roitman, M. F. (2011). Primary food reward and reward-predictive stimuli evoke different patterns of phasic dopamine signaling throughout the striatum. Eur. J. Neurosci. 34, 1997-2006. doi: 10.1111/j.1460-9568.2011.07914.x

Cacciapaglia, F., Saddoris, M. P., Wightman, R. M., and Carelli, R. M. (2012). Differential dopamine release dynamics in the nucleus accumbens core and shell track distinct aspects of goal-directed behavior for sucrose. Neuropharmacology 62, 2050-2056. doi: 10.1016/j.neuropharm.2011.12.027

Cadoni, C., and Di Chiara, G. (1999). Reciprocal changes in dopamine responsiveness in the nucleus accumbens shell and core and in the dorsal caudate-putamen in rats sensitized to morphine. Neuroscience 90, 447-455. doi: 10.1016/S03064522(98)00466-7

Cohen, T. E., Kaplan, S. W., Kandel, E. R., and Hawkins, R. D. (1997). A simplified preparation for relating cellular events to behavior: mechanisms contributing to habituation, dishabituation, and sensitization of the Aplysia gill-withdrawal reflex. J. Neurosci. 17, 2886-2899.

Corbit, L. H., and Balleine, B. W. (2011). The general and outcome-specific forms of Pavlovian-instrumental transfer are differentially mediated by the nucleus accumbens core and shell. J. Neurosci. 31, 11786-11794. doi: 10.1523/JNEUROSCI.2711-11.2011

Delmonico, R. L., Hanley-Peterson, P., and Englander, J. (1998). Group psychotherapy for persons with traumatic brain injury: management of frustration and substance abuse. J. Head Trauma Rehabil. 13, 10-22. doi: 10.1097/00001199199812000-00004

De Luca, M. A., Bimpisidis, Z., Bassareo, V., and Di Chiara, G. (2011). Influence of morphine sensitization on the responsiveness of mesolimbic and mesocortical dopamine transmission to appetitive and aversive gustatory stimuli. Psychopharmacology 216, 345-353. doi: 10.1007/s00213-011-2220-9

De Luca, M. A., Solinas, M., Bimpisidis, Z., Goldberg, S. R., and Di Chiara, G. (2012). Cannabinoid facilitation of behavioral and biochemical hedonic taste responses. Neuropharmacology 63, 161-168. doi: 10.1016/j.neuropharm.2011.10.018
Di Chiara, G. (1995). The role of dopamine in drug abuse viewed from the perspective of its role in motivation. Drug Alcohol Depend. 38, 155. doi: 10.1016/0376-8716(95)01164-T

Di Chiara, G., and Bassareo, V. (2007). Reward system and the brain: what dopamine does and doesn't do. Curr. Opin. Pharmacol. 7, 69-76. doi: 10.1016/j.coph.2006.11.003

Di Chiara, G., Bassareo, V., Fenu, S., De Luca, M. A., Spina, L., Cadoni, C., et al. (2004). Dopamine and drug addiction: the nucleus accumbens shell connection. Neuropharmacology 47, 227-241. doi: 10.1016/j.neuropharm. 2004.06.032

Di Chiara, G., and Imperato, A. (1988). Drugs abused by humans preferentially increase synaptic dopamine concentrations in the mesolimbic system of freely moving rats. Proc. Natl. Acad. Sci. U.S.A. 85, 5274-5278. doi: 10.1073/pnas.85.14.5274

Fibiger, H. C., and Phillips, A. G. (1988). Mesocorticolimbic dopamine systems and reward. Ann. N. Y. Acad. Sci. 537, 206-215. doi: 10.1111/j.17496632.1988.tb42107.x

Goldstein, R. Z., and Volkow, N. D. (2011). Dysfunction of the prefrontal cortex in addiction: neuroimaging findings and clinical implications. Nat. Rev. Neurosci. 12, 652-669. doi: 10.1038/nrn3119

Granon, S., Passetti, F., Thomas, K. L., Dalley, J. W., Everitt, B. J., and Robbins, T. W. (2000). Enhanced and impaired attentional performance after infusion of D1 dopaminergic receptor agents into rat prefrontal cortex. J. Neurosci. 20, 1208-1215.

Jaskiw, G. E., Weinberger, D. R., and Crawley, J. N. (1991). Microinjection of apomorphine into the prefrontal cortex of the rat reduces dopamine metabolite concentrations in microdialysate from the caudate nucleus. Biol. Psychiatry 29, 703-706. doi: 10.1016/0006-3223(91)90144-B

Kalivas, P. W., and Volkow, N. D. (2005). The neural basis of addiction: a pathology of motivation and choice. Am. J. Psychiatry 162, 1403-1413. doi: 10.1176/appi.ajp.162.8.1403

Kennerley, S. W., and Walton, M. E. (2011). Decision making and reward in frontal cortex: complementary evidence from neurophysiological and neuropsychological studies. Behav. Neurosci. 125, 297-317. doi: 10.1037/a0023575

Khan, Z. U., and Muly, E. C. (2011). Molecular mechanisms of working memory. Behav. Brain Res. 219, 329-341. doi: 10.1016/j.bbr.2010.12.039

Lacroix, L., Broersen, L. M., Feldon, J., and Weiner, I. (2000). Effects of local infusions of dopaminergic drugs into the medial prefrontal cortex of rats on latent inhibition, prepulse inhibition and amphetamine-induced activity. Behav. Brain Res. 107, 111-121. doi: 10.1016/S0166-4328(99)00118-7

Lammel, S., Lim, B. K., Ran, C., Huang, K. W., Betley, M. J., Tye, K. M., et al. (2012). Input-specific control of reward and aversion in the ventral tegmental area. Nature 491, 212-217. doi: 10.1038/nature11527

Louilot, A., Le Moal, M., and Simon, H. (1989). Opposite influences of dopaminergic pathways to the prefrontal cortex or the septum on the dopaminergic transmission in the nucleus accumbens. An in vivo voltammetric study. Neuroscience 29, 45-56. doi: 10.1016/0306-4522(89)90331-X

McCutcheon, J. E., Ebner, S. R., Loriaux, A. L., and Roitman, M. F. (2012). Encoding of aversion by dopamine and the nucleus accumbens. Front. Neurosci. 6:137. doi: $10.3389 /$ fnins. 2012.00137

Murase, S., Grenhoff, J., Chouvet, G., Gonon, G. G., and Svensson, T. H. (1993). Prefrontal cortex regulates burst firing and transmitter release in rat mesolimbic dopamine neurons studies in vivo. Neurosci. Lett. 157, 53-56. doi: 10.1016/03043940(93)90641-W

Pontieri, F. E., Tanda, G., and Di Chiara, G. (1995). Intravenous cocaine, morphine, and amphetamine preferentially increase extracellular dopamine in the "shell" as compared with the "core" of the rat nucleus accumbens. Proc. Natl. Acad. Sci. U.S.A. 92, 12304-12308. doi: 10.1073/pnas.92.26.12304

Pontieri, F. E., Tanda, G., Orzi, F., and Di Chiara, G. (1996). Effects of nicotine on the nucleus accumbens and similarity to those of addictive drugs. Nature 382, 255-257. doi: 10.1038/382255a0

Rankin, C. H., Abrams, T., Barry, R. J., Bhatnagar, S., Clayton, D. F., and Colombo, J. (2009). Habituation revisited: an updated and revised description of the behavioral characteristics of habituation. Neurobiol. Learn. Mem. 92, 135-138. doi: 10.1016/j.nlm.2008.09.012

Redgrave, P., Prescott, T. J., and Gurney, K. (1999). Is the short-latency dopamine response too short to signal reward error? Trends Neurosci. 22, 146-151. doi: $10.1016 /$ S0166-2236(98)01373-3 
Robbins, T. W. (2002). The 5-choice serial reaction time task: behavioural pharmacology and functional neurochemistry. Psychopharmacology 163, 362-380. doi: 10.1007/s00213-002-1154-7

Schenk, S., Horger, B. A., Peltier, R., and Shelton, K. (1991). Supersensitivity to the reinforcing effects of cocaine following 6-hydroxydopamine lesions to the medial prefrontal cortex in rats. Brain Res. 543, 227-235. doi: 10.1016/00068993(91)90032-Q

Schultz, W. (1998). Predictive reward signal of dopamine neurons. J. Neurophysiol. $80,1-27$.

Seamans, J. K., and Yang, C. R. (2004). The principal features and mechanisms of dopamine modulation in the prefrontal cortex. Prog. Neurobiol. 74, 1-58. doi: 10.1016/j.pneurobio.2004.05.006

Sullivan, R. M. (2004). Hemispheric asymmetry in stress processing in rat prefrontal cortex and the role of mesocortical dopamine. Stress 7, 131-143. doi $10.1080 / 102538900410001679310$

Taber, M. T., and Fibiger, H. C. (1995). Electrical stimulation of the prefrontal cortex increases dopamine release in the nucleus accumbens of the rat: modulation by metabotropic glutamate receptors. J. Neurosci. 15, 3896-3904.

Tanda, G., Pontieri, F. E., and Di Chiara, G. (1997). Cannabinoid and heroin activation of mesolimbic dopamine transmission by a common $\mu 1$ opioid receptor mechanism. Science 276, 2048-2050. doi: 10.1126/science.276.5321.2048

Thompson, R. F., and Spencer, W. A. (1966). Habituation: a model phenomenon for the study of neuronal substrates of behavior. Psychol. Rev. 73, 16-43. doi: $10.1037 / \mathrm{h} 0022681$

Van den Oever, M. C., Spijker, S., Smit, A. B., and De Vries, T. J. (2010). Prefrontal cortex plasticity mechanisms in drug seeking and relapse. Neurosci. Biobehav. Rev. 35, 276-284. doi: 10.1016/j.neubiorev.2009.11.016
Vezina, P., Blanc, G., Glowinski, J., and Tassin, J. P. (1991). Opposed behavioural outputs of increased dopamine transmission in prefrontocortical and subcortical areas: a role for the cortical D-1 dopamine receptors. Eur. J. Neurosci. 10, 10011007. doi: 10.1111/j.1460-9568.1991.tb00036.x

Weissenborn, R., Robbins, T. W., and Everitt, B. J. (1997). Effects of medial prefrontal or anterior cingulate cortex lesions on responding for cocaine under fixed-ratio and second order schedules of reinforcement in rats. Psychopharmacology (Berl.) 134, 242-257. doi: 10.1007/s002130050447

Westerink, B. H. (1995). Brain microdialysis and its application for the study of animal behaviour. Behav. Brain Res. 70, 103-124. doi: 10.1016/01664328(95)80001-8

Conflict of Interest Statement: The author declares that the research was conducted in the absence of any commercial or financial relationships that could be construed as a potential conflict of interest.

Received: 14 October 2013; accepted: 13 February 2014; published online: 04 March 2014.

Citation: De Luca MA (2014) Habituation of the responsiveness of mesolimbic and mesocortical dopamine transmission to taste stimuli. Front. Integr. Neurosci. 8:21. doi: 10.3389/fnint.2014.00021

This article was submitted to the journal Frontiers in Integrative Neuroscience. Copyright (C) 2014 De Luca. This is an open-access article distributed under the terms of the Creative Commons Attribution License (CC BY). The use, distribution or reproduction in other forums is permitted, provided the original author(s) or licensor are credited and that the original publication in this journal is cited, in accordance with accepted academic practice. No use, distribution or reproduction is permitted which does not comply with these terms. 